other side to ascend during a deep inspiration. 2. A lowering of the upper line of resonance in whole or in part as compared with that on the opposite side. Posteriorly, for instance, the line may occupy the position shown in Fig. 2 (right side), or the fall may be evident only at the anterior extremity, or it may be general all along the line. In estimating this a slight allowance is to be made in favour of the left side but only in that portion of it anterior to the vertebral segment. It should also be ascertained that the line on one side is not abnormally high from emphysema. 3. Indistinct definition of the upper or outer lines of resonance-the resonant and dull areas merging more or less into each other. 4 Inward displacement of the outer line of resonance. Should one or more of these signs be present it may be possible to detect some deficiency in the excursion of the diaphragm on the same side.

The results obtained by percussion are next compared with those of palpation and auscultation. The points for consideration are chiefly these: 1 . The breath sounds disproportionately feeble in one or other of the anterior segments (especially if the right side approximates to the left). 2. Any approximation in intensity in the vocal resonance on the left side to that on the right, either generally or in one or other segment. 3. The presence of whispering resonance and expiratory breath sound over the middle or posterior segment on the left side, noting specially the pitch and duration of expiration ; if present on quiet breathing, and comparing with the same segment on the opposite side. 4. A discrepancy in the characters of the physical signs over different parts of the same segment; as already mentioned, the signs over each segment should be uniform throughout. 5 Encroachment of the signs pertaining to one segment or another-applying more particularly to the left side, as, for instance, bronchial breathing and bronchophony extending beyond the margin of the vertebral segment.

It is most usual when examining the apices in this way to find any abnormality with regard to the lines of resonance existing alongside some change in the normal character of a segment or segments : thus immobility of the upper line of resunance may coexist with weak breath sounds in the anterior segment, or a fall in the highest part of the line on the left side with the presence of expiration sound and whispering resonance in the middle segment. On the right side the results of percussion are more especially valuable, as the auscultatory signs by themselves are less definite there. We note that the delimitation of the percussion areas may be verified by (1) the vocal and whispering resonances, and (2) "transsonance." A stethoscope with a small chest-piece (three-quarters of an inch in diameter) should be used. ${ }^{2}$ It is moved along the segments in such a manner that each successive application partially overlaps the last. At the limits of resonance the sounds will change. "Transsonance" is a form of auscultatory percussion which is recommended by Abrams. A bone, such as a vertebra or the manubrium sterni, is directly struck with the finger or a hammer while the stethoscope is moved along the parts to be demarcated, the same point being struck all the while. A change of tone occurs in passing to a part of different density. By " transsonance" we may also compare one apex with another.

It has been pointed out that the auscultatory phenomena over the right apex are less trustworthy, but in addition to the aids just described there is another not to be lost sight of. It is, that in cases of disease the difference in the expiration sound in quiet and deep breathing respectively is much less marked than in health. In disease the bronchial type may be equally well marked in both forms of breathing. This is a feature which is of general application.

Occasionally in healthy lungs one hears a few crepitations over the apex (usually in the anterior segment) which are atelectatic in character. They only occur in the end of the first deep inspirations taken, and disappear completely after, it may be, one or two inspirations. They differ in character from those due to tubercle, being also more numerous. "Tuberculous crepitation, on the other hand, is always persistent.

It sometimes happens that a tuberculous apex becomes hyper-resonant, either from local emphysema or relaxation of the pulmonary tissue. A difference in the position or in the capability of movement of the line of resonance may

2 Obtainable by unscrewing the ivory chest-piece of the flexible Binaural stethoscope. then be found on that side, and other differences may be detected in the normal relationships of the auscultatory signs over the segments. It is very easy to diagnose a healed tuberculous focus in the apex by this method.

Although little else has been attempted in this communication than to outline the technique employed, its successful application in individual cases is entirely a matter of practice and, it may be added, of patience.

Heurietta-street, $\mathbf{W}$.

\section{A RARE FORM OF PURPURA.}

\section{By H. LAING GoRdon, M.D., C.M. Edin.}

THE case described below offers, in the present state of knowledge, little more than a pretty subject for academic discussion ; but an account of this unusual variety of an uncommon form of disease may supply useful facts or hints to investigators of the nature of purpura.

It does not appear that so far the clinical appearances of the disease or pathological investigation have demonstrated its origin, although they have stimulated ingenuity in grouping and classification. There will be no small difficulty in fitting this case into one particular group. The term "purpura," indeed, describes it inadequately, while to call it "Henoch's purpura" is like putting a fat cache-pot on a narrow shelf. The bestowal of Henoch's name on a rare group of cases gives them a fictitious importance; the symptoms he described as "added to purpura and articular swellings "-viz., vomiting, intestinal hæmorrhage, and colic -are probably due to internal extravasations of blood; and if this be so, "Henoch's purpura" is revealed as a severe form of ordinary purpura. The necropsy on Silbermann's patient referred to in the article "Purpura" in Allbutt's "System of Medicine" 1 tends to confirm this view. Henoch himself wrote only of "Eine eigenthümliche Form von Purpura," using an adjective which might qualify every case of purpura described since he wrote. The case to be narrated exhibited the symptoms described by Henoch, added to purpura and articular swelling, but had other severe symptoms superadded which did not appear to be attributable to extravasation. An opinion on the origin of a group of symptoms must be, failing definite evidence, a mere individual impression. Dogmatism is a snare but deprecation is craven, and so, in the absence of any specific organism to spare us the use of vague terms, my impression would ticket this case as either "nervous" or "rheumatic."

The patient, a boy, aged 14 years, known to me as being nervous and abnormally sensitive, persevering at work and plucky in games, was taken ill on Feb. 27th, 1901, complaining of pain and stiffness in the limbs and a rash on the legs. These symptoms disappeared in the morning but returned the next evening with increased severity, so that on March 1st the head-master of the boarding-school in which the boy was a pupil asked me to see him. There was no history of previous rheumatism or of any recent illness except slight sickness and transient earache on Feb. 23rd. I found the patient complaining of pain and tenderness in the knees, ankles, elbows, and wrists without heat or swelling. The temperature was normal and the pulse was 78 ; a slight fur was present on the tongue. The extensor surfaces of both legs, the inner surface of the thighs, and the outer aspect of the elbows were dotted thickly with a purpuric rash, some spots being red, unraised, and recent, others having begun already to fade, while all were indifferent to pressure. The diagnosis of "purpura rheumatica" seemed to be clear, but the same evening (March 1st) the onset of colic and hæmorrhage from the bowels proved it to be premature. In 24 hours over a pint of blood was lost; happily this symptom did not recur. On the 2nd and 3rd there was no more purpura, but on the 3rd the anterior aspect of each ankle was a mass of urticaria, while the pain was moving from joint to joint. The dorsum of each foot was tender and enormously swollen on the 2nd, but on the 3rd this had disappeared completely, giving place to similar conditions of the elbows and wrists. On the 3 rd the colic also returned with repeated nausea and vomiting of bile; the abdomen was tender all over. The patient's helplessness on the same day was increased by sudden tenderness 
and pain in both temporal regions rapidly followed by cedematous swellings of the size of an orange; the next day there was no trace of these swellings beyond soreness at their site.

From this date (the 3rd) there followed a rich abundance and variety of symptoms. From hour to hour, not merely from day to day, the clinical picture would change in a manner which defies description but which soon reduced the boy's attendants to a state of stolid but sympathetic expectancy and the patient himself to complete indifference. He seldom spoke throughout his long illness and even his monosyllabic utterances were accompanied by tears. In the course of 24 hours ugly swellings would appear and disappear, a dozen joints would be racked with pain in rapid succession, and the surface of the body would exhibit a whole atlas of skin diseases. Simple erythema and purpura, urticaria, and erythema exudativum in all its varieties, cropped out and disappeared for week after week in disheartening fashion; blushes and spots, stony nodes and nodules, and enormous papules presenting themselves and vanishing one after another or in groups, but always irregularly in order and seat.

On the 4th there was occasional colic; on the 5th the most prominent features were cedema of the right upper eyelid of the size of a hen's egg, and in the evening return of the colic and vomiting, and severe pain in the elbows, wrists, and ankles, while both cheeks swelled suddenly, and erythema nodosum and purpura contended for supremacy in the limbs. Purpura was triumphant on the morning of the 6th and the cdema was transferred from the cheeks to the forehead as though a variety of grotesque distortions of the features were the object ; in the afternoon the buttocks were covered thickly with erythema papulatum. But the colic remained the most anxious symptom up to March 8th when it subsided in favour of pain all over the back, and this gave place in turn to pain, œdema, and tenderness of the left side of the trunk, the left thigh, knee and hand, and both legs, causing inability to move. The patient was able to move comfortably once more by 9.30 P. M. On the 9 th and he had no swelling save over one knee and one shoulder, and he was able to sleep well. At 1 A.M. on the 10th he awoke with great abdominal pain and vomiting resulting in an alarming collapse which caused several anxious hour and required active measures. By midday the colic had gone, but a copious purpuric rash had appeared on the face and elbows. From the 12th to the 15th the colic decreased the flying pains and swellings continued until the 13th, and the only fresh rash was purpuric on both cheeks, the left knee, arm, and hand. The 14th was the first day that could be called satisfactory; the patient felt better, there were no pains or swellings, and the skin affection was limited to small crops of purpura. At 8 A.M. on the 15th he was desquamating "all over," but by 1 P.M. his arms were thickly covered with purpura from shoulder to wrist. From this date until the 19th no new features arose, although the old symptoms of pain, swelling, and rash rang the changes on the joints and the different areas of the body, but with less vigour. On the 19th for a brief period there were no pain, no colic, no new rash, a clean tongue, and a normal temperature in the patient, and in his attendants some hope of permanent improvement, when suddenly his flushed face drew attention to the pulse and temperature. Without apparent cause the pulse had risen to 104 and the temperature to $99 \cdot 4^{\circ} \mathrm{F}$. ; there were no abnormal cardiac signs. Up to this time the pulse had been the one cheerful feature, steady at 80 and rarely up to 90 , and of good character, while the temperature had never exceeded $99^{\circ}$. In the next few days the temperature rose slowly to $100 \cdot 4^{\circ}$ of an evening and $100^{\circ}$ in the morning, while the pulse-rate varied day and night between 120 and 130 . On the 22 nd the old symptoms took a new lease, colic and purpura, urticaria, cedema, and erythema, sudden and vanishing pains and swellings, once more perplexing us. On the 27th it was noted that the pains had left the region of the great joints and were affecting only the hands and feet, the axilla and popliteal spaces, and the muscles of all the limbs. On the 28th there was actually no pain, swelling, or rash, only vigorous desquamation once more; but the increased pulserate and temperature warned us not to draw encouragement again from the suspension of the original symptoms. The patient had a restless night; at $11 \mathrm{~A} . \mathrm{M}$. on the 29 th the pulse was 136 and the temperature was $99.4^{\circ}$ and for the first time a loud systolic murmur was detected in the mitral area without any increase of cardiac dulness. The cardiac condition now became the chief anxiety; there was, from this time, no return of the original symptoms except that the skin eruptions, especially the purpura, recurred every night for several weeks. It was interesting to observe that an erythematous blush could be produced at any time on any portion of the chest and abdomen simply by letting the patient know that one's attention was directed to that portion. The pulse-rate fell slowly to about 106 by April 26th, on which day the temperature reached normal. The cardiac dulness remained unaffected. By May 4th the pulse was under 100 and on the 27th he was able to watch the school sports from his window, although he paid for the pleasure by a large crop of purpura on his legs and a slightly raised temperature-90 days after the onset of his illness; but small crops of purpura were not uncommon five months after the date of onset of his illness. The convalescence was marked by a gradual lessening in intensity of the cardiac murmur, encouraging a hope that it would ultimately disappear; but once more a new development retarded recovery. Scarcely had he begun to use his legs when distinct, but happily not severe, chorea set in, the head, neck, and upper extremities, as well as the face, being affected mostly. This condition persisted for several months but did not become serious; indeed, the boy was able to make a voyage to South Africa during the winter. I have been unable to obtain a report as to his present condition but he is said to be well although " nervous" as before.

During the illness examination of the blood gave no information. Frequent examinations of the urine detected no renal mischief. During the first three weeks of the illness the total daily flow of urine was diminished more than there appeared reason for ; it averaged, during that period, 18 ounces and ranged from nil to 29 ounces per diem. No benefit was obtained from the use of salicylates or any of the numerous drugs selected out of recommended remedies. Henoch's recommendation of icebags to the abdomen for the colic was of no avail. Careful nursing and feeding, together: with palliative treatment of symptoms as they arose-a proceeding which often recalled the carpenter's estimate of the prospects of the seven maids with seven mops-was the method of treatment employed in this case.

No doubt the case presented a curious jumbling-up of the symptoms of "Henoch's purpura" and "purpura rheumatica" with additions. The transient earache on Feb. 23rd has. been suggested as a possible source of septic infection. The suggestion is incapable of proof or disproof. Whatever the poison it appeared to act upon the nervous system.

The case differed from those described by Henoch as

follows : (1) In its more continuous nature ; there were no "intervals of several days or weeks or even of a year" (Henoch); (2) in the prominence of skin eruptions other than purpura, and of what can only be called "flying oedema" (3) in the cardiac complication ${ }^{2}$; (4) in the chorea; and (5) in the diminished daily flow of urine.

I had the advantage of meeting Dr. H. P. Hawkins in consultation over this case. Florence.

\section{A CASE OF APPENDICITIS WITH GENERAL PERITONITIS.}

BY W. GREENWOOD SUTCLIFFE, F.R.C.S. ENG., HONORARY SURGEON TO MARGATE COTTAGE HOSPITAL AND THE ROYAL SEA-BathiNg hospitaI, MaRgate.

RECOVERY of cases of this gravity being sufficiently rare as compared with the small mortality attending opera. tions where the damaged part is only slightly shut off, the details of this case may be worth putting on record.

The case appears to have begun on Oct. 24th, 1902, the patient, a girl, aged 20 years, being seized on that night with violent abdominal pain which was treated in the usual domestic manner without medical advice by the application of hot fomentations, with the result that the pain subsided next day, and a similar attack having occurred 12 months before which had cleared up satisfactorily it was thought that the trouble was practically at an end. The patient was able partially to go on with her housework she, however, remained constipated and slight pains in the

Compare a case of purpura described by Dr. C. W. Buckley The Lancet, July 20th, 1901, p. 132 